Draft Version OCtOBER 24, 2018

Preprint typeset using $\mathrm{LAT}_{\mathrm{E}} \mathrm{X}$ style emulateapj v. 08/22/09

\title{
DISTRIBUTION AND KINEMATICS OF THE HCN(3-2) EMISSION DOWN TO THE INNERMOST REGION IN THE ENVELOPE OF THE O-RICH STAR W HYA
}

\author{
SÉbastien Muller ${ }^{1}$, Dinh-V-Trung ${ }^{1,2}$, Jin-Hua He ${ }^{1,3}$ \& Jeremy Lim ${ }^{1}$ \\ Draft version October 24, 2018
}

\begin{abstract}
We report high angular resolution observations of the HCN (3-2) line emission in the circumstellar envelope of the O-rich star W Hya with the Submillimeter Array. The proximity of this star allows us to image its molecular envelope with a spatial resolution of just $\sim 40 \mathrm{AU}$, corresponding to about 10 times the stellar diameter. We resolve the HCN (3-2) emission and find that it is centrally peaked and has a roughly spherically symmetrical distribution. This shows that HCN is formed in the innermost region of the envelope (within $\sim 10$ stellar radii), which is consistent with predictions from pulsationdriven shock chemistry models, and rules out the scenario in which HCN forms through photochemical reactions in the outer envelope. Our model suggests that the envelope decreases steeply in temperature and increases smoothly in velocity with radius, inconsistent with the standard model for mass-loss driven by radiative pressure on dust grains. We detect a velocity gradient of $\sim 5 \mathrm{~km} \mathrm{~s}^{-1}$ in the NW-SE direction over the central $40 \mathrm{AU}$. This velocity gradient is reminescent of that seen in $\mathrm{OH}$ maser lines, and could be caused by the rotation of the envelope or by a weak bipolar outflow.
\end{abstract}

Subject headings: circumstellar matter — radio lines: stars — stars: individual (W Hya) — stars: late-type — stars: winds, outflows

\section{INTRODUCTION}

The detection at radio frequencies of various molecules in the circumstellar envelopes of evolved stars has considerably improved our understanding of circumstellar chemistry. This is particularly true for the prototype of carbon stars, IRC +10216 , on which much attention has been focused. Several dozens of molecules have been detected in its circumstellar envelope (e.g., Cernicharo et al. 2000), reflecting its complex and rich chemistry. The inventory of molecules together with high angular resolution images of their spatial distribution have led to the construction of sophisticated chemical models for the circumstellar envelope of IRC +10216 (e.g., Glassgold et al. 1987; Millar \& Herbst 1994) that now form the basis of our understanding of the chemistry in C-rich envelopes.

The chemistry in O-rich envelopes, on the other hand, was not expected to be as rich because equilibrium chemistry calculations indicate that nearly all the carbon should be locked into $\mathrm{CO}$ and nitrogen into $\mathrm{N}_{2}$ (Tsuil 1973). Indeed, before 1985, the inventory of molecules detected around oxygen stars was sparse and did not include carbon-bearing molecules, except of CO. Deguchi \& Goldsmith (1985) reported the first detection of HCN towards O-rich stars. The list of HCN detections was later extended by Jewell et al. (1986), Lindqvist et al. (1988), Nercessian et al. (1989), Olofsson et al. (1998) and Bieging et al. (2000). Recently, Ziurys et al. (2007) also emphasized the chemical complexity in the envelope of the O-rich supergiant star

\footnotetext{
Electronic address: muller, trung, jhhe, jlim @asiaa.sinica.edu.tw

1 Academia Sinica, Institute of Astronomy and Astrophysics (ASIAA), P.O. Box 23-141, Taipei 106, Taiwan

2 On leave from Institute of Physics, Vietnamese Academy of Science \& Technology, 10, Daotan, BaDinh, Hanoi, Vietnam

3 New address: National Astronomical Observatories/Yunnan Observatory, Chinese Academy of Sciences, PO Box 110, Kunming, Yunnan Province 650011, China
}

VY CMa, following the detection of various molecules such as $\mathrm{HCO}^{+}$, CS, $\mathrm{NaCl}, \mathrm{PN}$ and $\mathrm{SiS}$.

Two competing models have been proposed to account for the formation of $\mathrm{HCN}$ in O-rich envelopes: photochemical reactions in the outer envelope (Charnley et al. 1995), or gas-phase non-equilibrium chemical reactions in the inner region close to the stellar photosphere (within few tens of $\mathrm{AU}$ from the star) due to shocks driven by stellar pulsation (Duari et al. 1999; Duari \& Hatchell 2000). The two models predict very different HCN spatial distribution: in a hollow shell-like structure of radius 200 to 1000 AU depending on the mass loss for photochemical reactions, or a centrally concentrated distribution extending up to the photodissociation radius of $\mathrm{HCN}$ for the shock-driven chemical reactions.

Current observational data seem to favor pulsationdriven shock chemistry formation of $\mathrm{HCN}$ in O-rich stars. Bieging et al. (2000) conducted single-dish observations toward a sample of 16 O-rich stars in the HCN (3-2) and (4-3) lines. The detection of these high density tracers is inconsistent with photochemical production of $\mathrm{HCN}$ in the outer envelope. The detection of the HCN (3-2) $\left(0,1^{1 c}, 0\right)$ and $(8-7)$ transitions toward $\chi$ Cyg gave further support for the formation of $\mathrm{HCN}$ in the innermost region of the envelope, within $\sim 20$ stellar radii (i.e. $\sim 30$ AU), based on excitation arguments (Duari \& Hatchell 2000). Marvel (2005) reported the first interferometric observations of $\mathrm{HCN}$ in the circumstellar envelopes of the O-rich stars IK Tau and TX Cam. In both stars, the HCN emission appears to be largely concentrated within $\sim 800 \mathrm{AU}$, as predicted by shock chemistry models.

If $\mathrm{HCN}$ indeed forms close to the stellar photosphere, it should be an excellent tracer of the inner envelope, and perhaps even of the wind acceleration zone where, in the standard model, dust particles form and radiation pressure on dust becomes most effective. Maser (e.g., $\mathrm{H}_{2} \mathrm{O}$ or $\mathrm{OH}$ ) emission in the inner envelope can be observed 
with very long baseline interferometry, but traces only a limited radial range. On the other hand, there are so far only few reports of high angular resolution observations of other thermal lines in the inner envelope, such as $\mathrm{SiO}(2-1) \nu=0$ (Lucas et al. 1992; Sahai \& Bieging 1993; Schöier et al. 2004), none of them with angular resolution higher than $\sim 100 \mathrm{AU}$.

In this letter, we report very high spatial resolution ( $40 \mathrm{AU}$ ) observations of the HCN (3-2) emission toward W Hya with the Submillimeter Array. W Hya is one of the closest O-rich stars at a distance of $78 \mathrm{pc}$ (based on Hipparcos data, Perryman et al. 1997, revised by Knapp et al. 2003), and shows the brightest HCN (32 ) and (4-3) emission amongst the sample of O-rich stars observed by Bieging et al. (2000). It is therefore an excellent target in which to investigate the distribution and kinematics of $\mathrm{HCN}$ in the circumstellar envelope of an O-rich star.

\section{OBSERVATIONS}

We observed W Hya on 2008 April 13 and 15 with the Submillimeter Array ${ }^{4}$ (SMA) in its very extended configuration. All eight antennas were operating on the first day of our observations, and six on the second day. The zenith atmospheric opacity was $\sim 0.2$ at $225 \mathrm{GHz}$ during both nights, resulting in system temperatures that changed between 200 and $600 \mathrm{~K}$ depending on source elevation.

The heterodyne SIS receivers were tuned to the frequency of the HCN (3-2) transition at $265.886 \mathrm{GHz}$ in the lower side band. The correlator was configured to give a spectral resolution of $0.8125 \mathrm{MHz}$, which corresponds to a velocity resolution of $\sim 0.9 \mathrm{~km} \mathrm{~s}^{-1}$. The line-free channels in both the upper and lower side bands, spanning a total bandwidth of $\sim 3.8 \mathrm{GHz}$ at $1.1 \mathrm{~mm}$, were used to make a map of the continuum. The bandpass of the individual antennas was derived from the bright quasar 3C273. Flux calibration was derived by observing Titan and Callisto. The quasars 1334-127 and 1313-333, located within $16^{\circ}$ of $\mathrm{W}$ Hya, were observed every 15 min for complex gain calibration. Data reduction was done separately for both tracks using MIR/IDL. The calibrated visibilities were written out in FITS format, and then converted into GILDAS format for imaging purpose using MAPPING.

The projected baselines ranged from 22 to $510 \mathrm{~m}$. Natural weighting of the visibilities yielded a synthesized beam of $0.55^{\prime \prime} \times 0.40^{\prime \prime}$ at a position angle of $26^{\circ}$. We used the CLARK algorithm to deconvolve the image. For the $\mathrm{HCN}$ (3-2) channel map, we estimate the $1 \sigma \mathrm{rms}$ noise level to be $15 \mathrm{mJy} /$ beam in each $0.9 \mathrm{~km} \mathrm{~s}^{-1}$ channels. For the $1.1 \mathrm{~mm}$ continuum, the noise level is $3 \mathrm{mJy} / \mathrm{beam}$. The primary beam of the SMA antennas spans a FWHM of $46.5^{\prime \prime}$ at $266 \mathrm{GHz}$.

\section{RESULTS}

The HCN (3-2) channel maps are shown in Figure 1 together with the $1.1 \mathrm{~mm}$ continuum map. The molecular emission, which is clearly resolved, is centrally peaked

\footnotetext{
4 The Submillimeter Array is a joint project between the Smithsonian Astrophysical Observatory and the Academia Sinica Institute of Astronomy and Astrophysics and is funded by the Smithsonian Institution and the Academia Sinica.
}

and has a roughly spherically symmetrical distribution. The continuum emission is unresolved and has a total flux density of $270 \pm 15 \mathrm{mJy}$ at $1.1 \mathrm{~mm}$, consistent with the value of $280 \pm 30 \mathrm{mJy}$ obtained by van der Veen et al. (1995) with the James Clerk Maxwell Telescope. We estimate a stellar blackbody contribution of $225 \mathrm{mJy}$ based on the stellar parameters given in Table 1, and which therefore dominates the $1.1 \mathrm{~mm}$ continuum emission.

W Hya is known to have a very extended dusty envelope with a radius of $\sim 30^{\prime}$ (Hawkins 1990). The photodissociation radius of $\mathrm{HCN}$, however, is expected to be much smaller, of order of $1^{\prime \prime}$, based on computations by Olofsson et al. (1998) (see their Eq.2). From a Gaussian fit of the visibilities, we find that the HCN (3-2) emitting region has a radius at FWHM of about $0.6^{\prime \prime}$, comparable to the size measured by Lucas et al. (1992) for the $\mathrm{SiO}(2-1)$ emission. Comparing our data with that from single-dish observations by Bieging et al. (2000), we estimate that we recover $\gtrsim 70 \%$ of the total line intensity within $28^{\prime \prime}$ of the star. A spectrum of the HCN (3-2) line, extracted at the peak position of the emission, is shown in Figure 2. The peak brightness temperature of the line is $\sim 300 \mathrm{~K}$. The line profile is clearly nonsymmetric with respect to the systemic velocity of $\sim 40$ $\mathrm{km} \mathrm{s}^{-1}$ (Cernicharo et al. 1997).

A visual inspection of the channel maps reveals a weak velocity gradient in the NW to SE direction. A fit of the visibilities yields a velocity gradient of $\sim 5 \mathrm{~km} \mathrm{~s}^{-1}$ at a position angle of $125^{\circ}$ over the $0.5^{\prime \prime}$ central region. Position-velocity diagrams along this direction, as well as on a perpendicular axis, are shown in Figure 3.

\section{MODELING}

The peak brightness temperature, line profile and radial distribution of the HCN (3-2) gas provide very useful constraints on the wind velocity and the physical conditions of the gas in the vicinity of W Hya. We used the molecular excitation and radiative transfer code of Dinh-V-Trung \& Nguven-Q-Rieu (2000) to interpret our observations and other relevant data. We assumed a spherical symmetry and did not attempt to reproduce the weak velocity gradient. The model parameters are summarized in Table 1 and reproduce remarkably well our data (see Fig 2 \& 4).

In the inner region of the envelope, the HCN molecules are excited by collisions with $\mathrm{H}_{2}$ and by the absorption of stellar photons at $3 \mu \mathrm{m}$ (to $\nu_{3}=1$ vibrational state), $7 \mu \mathrm{m}$ (to $02^{0} 0$ vibrational state), and $14 \mu \mathrm{m}$ (to $01^{1} 0$ vibrational state). In the case of $\mathrm{W}$ Hya, where little absorption by dust is expected, the pumping route through the $\nu_{3}=1$ and $01^{1} 0$ states are the most important because of the strong stellar radiation field at the corresponding wavelengths and large transition rates. In our code, we take into account the vibrational ground state, $\left(01^{1} 0\right)$ and $\nu_{3}=1$ vibrational states, and include explicitly all hyperfine levels up to $J=15$. In W Hya, we find that the $\nu_{3}=1$ absorption band of HCN falls within the deep $\mathrm{H}_{2} \mathrm{O}$ absorption band (Justtanont et al. 2004). The water molecules responsible for this absorption band are hot and expected to be very close to the central star, presumably interior to the $\mathrm{HCN}$ emitting region. Thus, for the sake of simplicity, we reduced the stellar radiation field by $50 \%$ at $3 \mu \mathrm{m}$.

The mass loss rate of $\mathrm{W}$ Hya is still quite uncertain. 
Zubko \& Elitzur (2000) derived a mass loss rate of about $10^{-6} \mathrm{M}_{\odot} \mathrm{yr}^{-1}$ (scaled to the revised distance of $78 \mathrm{pc}$ ), while Justtanont et al. (2005) favored a much lower rate of $2-3 \times 10^{-7} \mathrm{M}_{\odot} \mathrm{yr}^{-1}$. We adopt here a value of $5 \times 10^{-7}$ $\mathrm{M}_{\odot} \mathrm{yr}^{-1}$. The abundance of HCN relative to molecular hydrogen predicted from non-equilibrium chemical models (Duari et al. (1999); Duari \& Hatchell (2000)) may vary between a few times $10^{-6}$ and a few times $10^{-5}$, depending on the shock velocity. We adopt a representative constant value of $10^{-6}$. We note, however, that this assumption leads to a very slight overestimate of the HCN (3-2) intensity in the outer part of the HCN envelope (see Fig (4). Most likely, the abundance of HCN is gradually decreasing with radius due to the photodissociation by external radiation field. We also assumed a local turbulent velocity of $1 \mathrm{~km} \mathrm{~s}^{-1}$ in the envelope.

We tried various expansion velocity laws and found that the relation $\mathrm{V}(\mathrm{r}) \propto \log (\mathrm{r})$, starting from $2 \mathrm{~km} \mathrm{~s}^{-1}$ at an inner radius of $\sim 10^{14} \mathrm{~cm}$ and reaching $7 \mathrm{~km} \mathrm{~s}^{-1}$ at a radius of $10^{16} \mathrm{~cm}$, produces a satisfactory fit to the HCN (3-2) line profile, and is consistent with $\mathrm{OH}$ maser observations of Szymczak et al. (1998). Other faster rising velocity laws, such as those prescribed by Deguchi \& Nguyen-Q-Rieu (1990) or Zubko \& Elitzur (2000), do not reproduce the rounded-top and asymmetric profile of HCN (3-2) as seen by the SMA (they actually produce double-peak profiles).

The rounded-top line profile indicates that the HCN (3-2) is optically thick. In addition, we find that the excitation temperature of the HCN (3-2) transition follows closely the kinetic temperature of the gas. Unless the wind is highly clumpy, resulting in small filling factor in the beam, the brightness temperature of the HCN (3-2) emission, resolved by the SMA, is therefore directly related to the temperature profile in the inner region. The very high temperature profile used by Deguchi \& Nguyen-Q-Rieu (1990) or the one designated as GS in Figure 3 of Zubko \& Elitzur (2000) are clearly inconsistent with our data. The shallower temperature profile derived by Zubko \& Elitzur (2000) also produces too high a brightness temperature for the HCN (3-2) line because the kinetic temperature is above $400 \mathrm{~K}$ within an inner region of $50 \mathrm{AU}$ in diameter, which is comparable to the SMA synthesized beam of $\sim 0.5^{\prime \prime}$. In our model, we need to use a steep temperature profile $\mathrm{T}(\mathrm{r})$ $\propto \mathrm{r}^{-1}$, starting with $\mathrm{T}_{0}=650 \mathrm{~K}$ at the base of the envelope. The predicted line profile is compared with that observed in Figure 2, Our model also provides a good match to the single-dish profiles of the $\mathrm{HCN}(3-2)$ and (4-3) lines observed by Bieging et al. (2000).

We note that for the $\mathrm{HCN}(3-2)$ line, the resulting profile is asymmetric and does not peak at the systemic velocity. That is due to the strong self-absorption arising from the combination of steep temperature profile and slow acceleration in the inner envelope. This effect can also explain the difference in the inferred systemic velocity from Gaussian fitting of optically thick lines such as $\mathrm{HCN}(4-3), \mathrm{SiO}(5-4)$ and (8-7) (Bieging et al. 2000), with respect to other lines such as $\mathrm{SiO}(2-1)$ and $\mathrm{CO}$ lines (Cernicharo et al. 1997).

\section{DISCUSSION AND PERSPECTIVES}

TABLE 1: MODEL PARAMETERS

\begin{tabular}{ll}
\hline \hline & \multicolumn{1}{c}{ STAR } \\
Distance & $\mathrm{D}=78 \mathrm{pc}$ \\
Systemic velocity (LSR) & $\mathrm{V}_{\mathrm{SYS}}=40.4 \mathrm{~km} \mathrm{~s}^{-1}$ \\
Effective temperature & $\mathrm{T}_{\text {eff, } \star}=2500 \mathrm{~K}$ \\
Stellar radius & $\mathrm{R}_{\star}=2.73 \times 10^{13} \mathrm{~cm}$ \\
& ENVELOPE \\
Inner radius & $\mathrm{R}_{\text {in }}=1 \times 10^{14} \mathrm{~cm}$ \\
Outer radius & $\mathrm{R}_{\text {out }}=3 \times 10^{15} \mathrm{~cm}$ \\
Mass-loss rate & $\dot{\mathrm{M}}=5 \times 10^{-7} \mathrm{M}_{\odot} \mathrm{yr}^{-1}$ \\
Temperature profile & $\left.\mathrm{T}(\mathrm{r})=650 \mathrm{~K}^{-1} / \mathrm{R}_{\mathrm{in}}\right)^{-1}$ \\
Launching velocity & $\mathrm{V}_{0}=2 \mathrm{~km} \mathrm{~s}^{-1}$ \\
Terminal velocity & $\mathrm{V}_{\infty}=7 \mathrm{~km} \mathrm{~s}^{-1}$ \\
Velocity law (r $<\mathrm{R}$ out $)$ & $\mathrm{V}(\mathrm{r})=\frac{\left(\mathrm{V}_{\infty}-\mathrm{V}_{0}\right) \log _{10}\left(\mathrm{r} / \mathrm{R}_{\text {in }}\right)}{\log _{10}\left(10^{16} \mathrm{~cm} / \mathrm{R}_{\text {in }}\right)}+V_{0}$ \\
Abundance of HCN & {$[\mathrm{HCN}] /\left[\mathrm{H}_{2}\right]=10^{-6}$} \\
Local turbulent velocity & $\sigma_{\text {turb }}=1 \mathrm{~km} \mathrm{~s}^{-1}$ \\
\hline
\end{tabular}

REFERENCES. - (1) Knapp et al. (2003); (2) Cernicharo et al. (1997); (3) Haniff et al. (1995); (4) Justtanont et al. (2005); (5) Miyoshi et al. (1994).

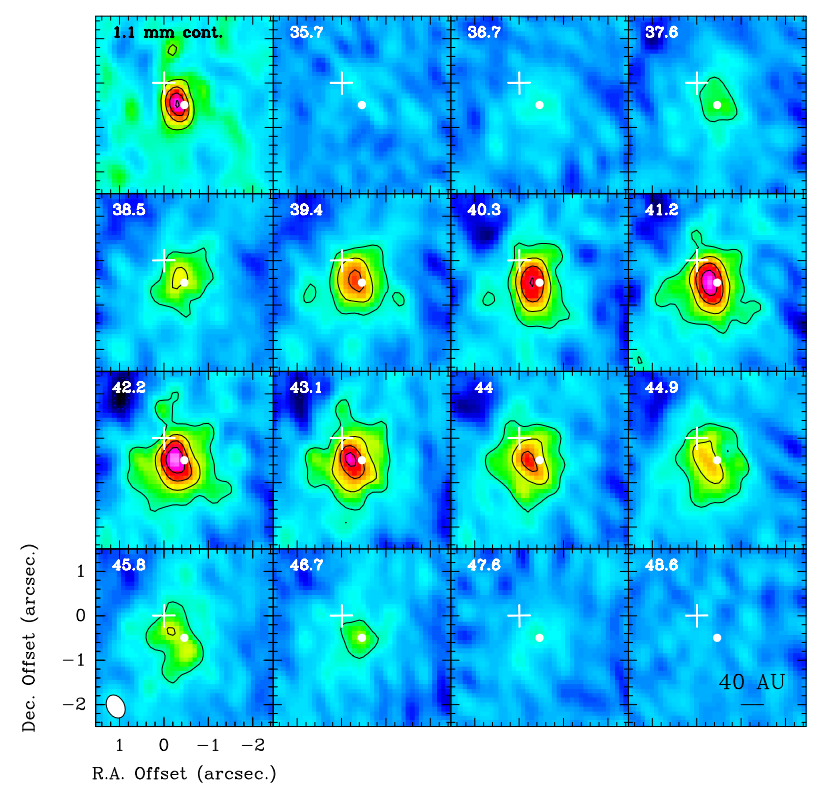

FIG. 1.- $1.1 \mathrm{~mm}$ continuum emission (top left corner box) and HCN (3-2) channel maps of W Hya as observed with the SMA very extended configuration. Contours are every $25 \mathrm{mJy} / \mathrm{beam}$ for the continuum map, and every $0.75 \mathrm{Jy} /$ beam $(5 \sigma)$, corresponding to $60 \mathrm{~K}$ for the synthesized beam of $0.55^{\prime \prime} \times 0.40^{\prime \prime}\left(\right.$ P.A. $\left.=26^{\circ}\right)$, for the channel maps. The white cross indicates the position of the phase center, set at the stellar position from the Hipparcos catalog in the year 2000 (R.A. $J 2000=13^{\mathrm{h}} 49^{\mathrm{m}} 01^{\mathrm{s}} .998$ and Dec. $J 2000=$ $\left.-28^{\circ} 22^{\prime} 03^{\prime \prime} 49\right)$. The white dot gives the expected position of the star at the date of our observations in 2008, due to its proper motion. The small offset $\left(\lesssim 0.15^{\prime \prime}\right)$ between this position and the peak emission may be due to baseline errors.

In the model proposed by Charnley et al. (1995), HCN is formed in the outer envelope as a result of photochemical reactions initiated from methane. The distribution of $\mathrm{HCN}$ is predicted to peak at a radius $\geq 200 \mathrm{AU}$ for a mass-loss rate $\geq 10^{-7} \mathrm{M}_{\odot} \mathrm{yr}^{-1}$. Our SMA observations, however, clearly reveal that in the case of W Hya, HCN is present, and thus should form, much closer to the stellar photosphere, within $20 \mathrm{AU}$. This is consistent with the shock chemistry model developed by Duari et al. (1999) and Duari \& Hatchell (2000). In this model, stellar pulsations induce strong and periodic shocks in a narrow region above the photosphere. Thermal equilibrium (TE) abundances are assumed as initial conditions in the pho- 


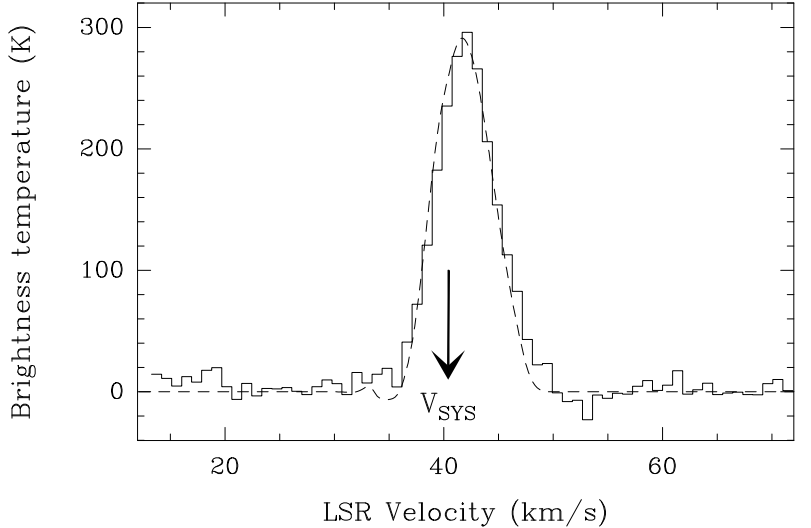

FIG. 2.- Brightness temperature at the peak of the HCN (3-2) emission as a function of velocity, with model prediction overlaid (dashed line). Note that the blue-shifted part of the spectrum is strongly affected by self-absorption.

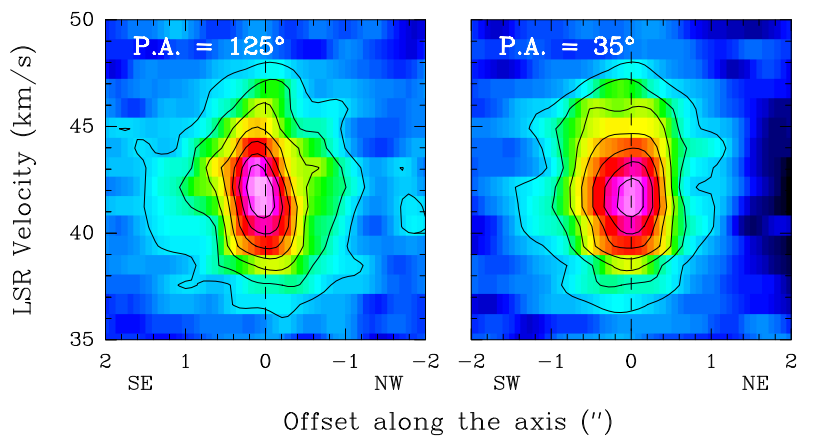

FIG. 3.- Position-velocity diagrams along two perpendicular axes. Note the small velocity gradient along the SE-NW direction.

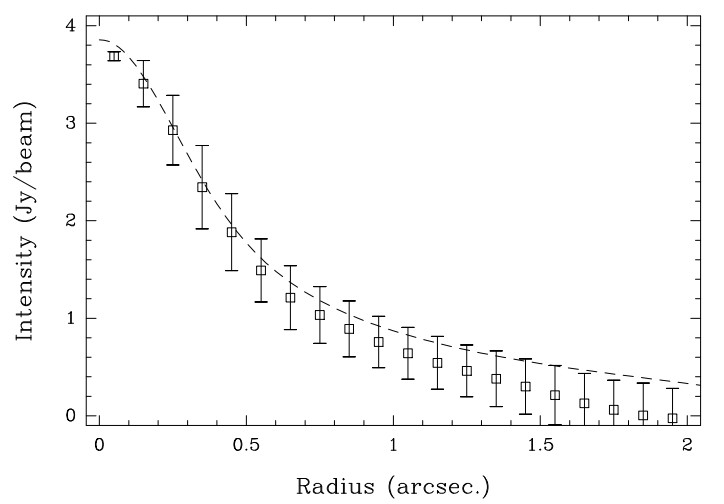

Fig. 4.- Azimuthal average of the HCN (3-2) emission as a function of radius at $\mathrm{V}_{\mathrm{LSR}}=42.1 \mathrm{~km} \mathrm{~s}^{-1}$, corresponding to the peak of the line profile in Figure 2 Our SMA data are indicated by open squares with error bars overlaid. The model prediction is shown in dashed line.

tosphere, and the evolution of chemical abundances is investigated in the post-shock region. As a net result of shock chemistry, several carbon-bearing species, such as $\mathrm{HCN}, \mathrm{CS}$ and $\mathrm{CO}_{2}$, are produced in significant amounts. Noticeably, the abundance of $\mathrm{HCN}$ can be increased by about 5 orders of magnitude with respect to its former $\mathrm{TE}$ value. The route to $\mathrm{HCN}$ is through the reaction of $\mathrm{CN}$ with $\mathrm{H}_{2}$, which is sensitive to temperature and becomes very efficient in the post-shock layer. Since HCN is chemically stable and does not participate in the formation of dust grains (unlike, e.g., $\mathrm{SiO}$ ) in the inner envelope, it further remains unaltered as it travels through the envelope, until it reaches the photodissociation region of the outer wind. As a result, $\mathrm{HCN}$ is a good tracer of the kinematics in the envelope.

The requirement of a smoothly increasing velocity with radius to reproduce the $\mathrm{HCN}(3-2)$ line profile for W Hya and maser observations (Szymczak et al. 1998) are inconsistent with a fast acceleration of the wind within $\lesssim 10$ stellar radii, as predicted by models of mass-loss driven by radiative pressure on dust grains (e.g., Kwok 1975; Goldreich \& Scoville 1976). Observations in other lines tracing different radii also called for a slow wind acceleration, which could be explained if dust grain formation occurs over large extent up to several hundreds of AU (Lucas et al. 1992), and/or if grain properties change through the envelope (Chapman \& Cohen 1986). Alternatively, the slow acceleration could result from the inefficiency of dust drag when the mass loss is small (see, e.g., Bowen 1988; Winters et al. 2000).

The velocity gradient along the SE-NW axis (see Fig (3) is about $5 \mathrm{~km} \mathrm{~s}^{-1}$ over $0.5^{\prime \prime}$. MERLIN observations of OH maser lines by Szymczak et al. (1998) reveal a remarkably similar trend in amplitude and position angle. Interestingly, Lattanzi et al. (1997) measured the visible diameter of $\mathrm{W}$ Hya along two orthogonal directions and found a significant asymmetry of $20 \%$, with elongation in a direction comparable to that of the velocity gradient seen in the envelope. It is not clear, however, whether this apparent elongation is due to some oblateness of the star or to a nonsymmetric brightness distribution over the stellar disk. The velocity gradient that we observe in the inner envelope of $\mathrm{W}$ Hya could be either due to the rotation of the envelope or to a weak bipolar outflow.

The next jump in angular resolution achievable with the Atacama Large Millimeter Array will allow us to probe the envelope even deeper, possibly down to the dust formation zone. Such observations could directly test the validity of shock chemistry models. In particular, very high angular resolution observations at different phases of the stellar variablity could directly show the effects of the pulsations on the formation of dust and molecules, and possible modulations of the mass loss (see, e.g., Diamond \& Kemball 2003).

\section{REFERENCES}

Bieging, J. H., et al., 2000, ApJ, 543, 897

Bowen, G. H., 1988, ApJ, 329, 299

Cernicharo, J., et al., 1997, A\&A, 319, 607

Cernicharo, J., Guélin, M. \& Kahane, C., 2000, A\&AS, 142, 181

Chapman, J. M. \& Cohen, R. J., 1986, MNRAS, 220, 513

Charnley, S. B., et al., 1995, MNRAS, 274, L53

Deguchi, S. \& Goldsmith, P. F., 1985, Nature, 317, 336

Deguchi, S. \& Nguyen-Q-Rieu, 1990, ApJ, 360, L27
Diamond, P. J. \& Kemball, A. J., 2003, ApJ, 599, 1372

Dinh-V-Trung \& Nguyen-Q-Rieu, 2000, A\&A, 361, 601

Duari, D., Cherchneff, I. \& Willacy, K., 1999, A\&A, 341, L47

Duari, D. \& Hatchell, J., 2000, A\&A, 358, L25

Glassgold, A. E., et al., 1987, A\&A, 180, 183

Goldreich, P. \& Scoville, N., 1976, ApJ, 205, 144

Haniff, C. A., Scholz, M. \& Tuthill, P. G., 1995, MNRAS, 276, 640 Hawkins, G. W., 1990, A\&A, 229, L5 
Jewell, P. R., et al., 1986, Nature, 323, 311 Justtanont, K., et al., 2004, A\&A, 417, 625 Justtanont, K., et al., 2005, A\&A, 439, 627 Knapp, G. R., et al., 2003, A\&A, 403, 993

Kwok, S., 1975, ApJ, 198, 583

Lattanzi, M. G., et al., 1997, ApJ, 485, 328 Lindqvist, M., et al., 1988, A\&A, 205, L15

Lucas, R., et al., 1992, A\&A, 262, 491

Marvel, K. B., 2005, AJ, 130, 261

Millar, T. J. \& Herbst, E., 1994, A\&A, 288, 561

Miyoshi, M., et al., 1994, Nature, 371, 395

Nercessian, E., et al., 1989, A\&A, 210, 225

Olofsson, H., et al., 1998, A\&A, 329, 1059
Perryman, M. A. C., et al., 1997, A\&A, 323, L49

Sahai, R. \& Bieging, J. H., 1993, AJ, 105, 595

Schöier, F. L., et al., 2004, A\&A, 422, 651

Szymczak, M., et al., 1998, MNRAS, 297, 1151

Tsuji, T., 1973, A\&A, 23, 411

van der Veen, W. E. C. J., et al., 1995, A\&A, 295, 445

Walmsley, C. M.; et al., 1991, A\&A, 248, 555

Winters, J. M., et al., 2000, A\&A, 361, 641

Ziurys, L. M., et al., 2007, Nature, 447, 1094

Zubko, V. \& Elitzur, M., 2000, ApJ, 544, L137 